\title{
On Two Problems for Matrix Polytopes
}

\author{
Şerife Yılmaz*, Taner Büyükköroğlu \\ Department of Mathematics, Faculty of Science, Anadolu University, Eskisehir, Turkey \\ Email: serifeyilmaz@anadolu.edu.tr, tbuyukkoroglu@anadolu.edu.tr
}

Received 21 July 2014; revised 20 August 2014; accepted 8 September 2014

Copyright (C) 2014 by authors and Scientific Research Publishing Inc.

This work is licensed under the Creative Commons Attribution International License (CC BY).

http://creativecommons.org/licenses/by/4.0/

(c) (i) Open Access

\section{Abstract}

We consider two problems from stability theory of matrix polytopes: the existence of common quadratic Lyapunov functions and the existence of a stable member. We show the applicability of the gradient algorithm and give a new sufficient condition for the second problem. A number of examples are considered.

\section{Keywords}

Stable Matrix, Matrix Family, Common Quadratic Lyapunov Functions, Switched System, Gradient Method

\section{Introduction}

Consider the switched system

$$
\dot{x}(t)=A x(t), \quad A \in\left\{A_{1}, A_{2}, \cdots, A_{N}\right\}
$$

where $\dot{x}(t) \in \mathbb{R}^{n}, t \geq 0$. In Equation (1), the matrix $A$ switches among $N$ matrices $A_{1}, A_{2}, \cdots, A_{N}$.

Switching signal $\sigma(t)$ is piecewise continuous from the right function $\sigma:[0, \infty) \rightarrow\{1,2, \cdots, N\}$ and the switching times are arbitrary. For the switched system (1) with initial condition $x(0)=x_{0}$ and with switching signal $\sigma(t)$ denotes the solution by $x\left(t, x_{0}, \sigma(\cdot)\right)$.

Definition 1. The origin is uniformly asymptotically stable (UAS) for the system (1) if for every $\varepsilon>0$ there exists $\delta>0$ such that for every signal $\sigma(t)$ and initial state $x_{0}$ with $\left\|x_{0}\right\|<\delta$, the inequality $\left\|x\left(t, x_{0}, \sigma(\cdot)\right)\right\|<\varepsilon$ is satisfied for all $t>0$ and uniformly on $\sigma(\cdot)$

$$
\lim _{t \rightarrow \infty} x\left(t, x_{0}, \sigma(\cdot)\right)=0 \text {. }
$$

If all systems in (1) share a common quadratic Lyapunov function (CQLF) $V(x)=x^{\mathrm{T}} P x$ then the switched

*Corresponding author. 
system is UAS ( $T$ denotes the transpose).

In this case there exists a common $P>0$ such that

$$
A_{i}^{\mathrm{T}} P+P A_{i}<0 \quad(i=1,2, \cdots, N)
$$

and $P$ is called a common solution to the set of Lyapunov matrix inequalities (2).

The problem of existence of common positive definite solution $P$ of (2) has been studied in a lot of works (see [1]-[9] and references therein). Numerical solution for common $P$ via nondifferentiable convex optimization has been discussed in [10].

In the first part of the paper, the problem of existence of CQLF is investigated by Kelley's method. This method is applied when CQLF problem is treated as a convex optimization problem.

Second part of the paper is devoted to the following question:

Let $B \subset \mathbb{R}^{l}$ be a compact, for $q \in B$ the matrix $A(q)$ is a real $n \times n$ matrix. Is there a Hurwitz stable member (all eigenvalues lie in the open left half plane) in the family

$$
\{A(q): q \in B\}
$$

or equivalently is there $q^{*} \in B$ such that $A\left(q^{*}\right)$ is stable? This problem is one of the hard and important problems in control theory (see [11]). Numerical solution of this problem is considered in [12]. In this paper we reduce this problem to a non-convex optimization problem.

\section{Common Quadratic Lyapunov Function}

For the switched system

$$
\dot{x}=\left\{A_{1}, A_{2}, \cdots, A_{N}\right\} x
$$

consider the problem of determination of CQLF $V(x)=x^{\mathrm{T}} P x$ where $P>0$. We are going to investigate it by Kelley's cutting-plane method. This method gives new sufficient condition (Theorem 2) and new algorithm (Algorithm 1) which is more effective in comparison with the algorithm from [10].

Consider the problem of existence of a common $P>0$ such that

$$
A_{i}^{\mathrm{T}} P+P A_{i}<0 \quad(i=1,2, \cdots, N) .
$$

Let $x \in \mathbb{R}^{r}$ be $x^{\mathrm{T}}=\left(x_{1}, x_{2}, \cdots, x_{r}\right)$ and $P$ be an $n \times n$ symmetric matrix defined as

$$
P=P(x)=\left(\begin{array}{cccc}
x_{1} & x_{2} & \cdots & x_{n} \\
x_{2} & x_{n+1} & \cdots & x_{2 n-1} \\
\vdots & \vdots & \ddots & \vdots \\
x_{n} & x_{2 n-1} & \cdots & x_{r}
\end{array}\right) \quad\left(r=\frac{n(n+1)}{2}\right)
$$

Define

$$
\phi(x)=\max _{1 \leq i \leq N} \lambda_{\max }\left(A_{i}^{\mathrm{T}} P+P A_{i}\right)=\max _{1 \leq i \leq N,\|u\|=1} u^{\mathrm{T}}\left(A_{i}^{\mathrm{T}} P+P A_{i}\right) u .
$$

If there exists $x_{*}$ such that $P\left(x_{*}\right)>0$ and $\phi\left(x_{*}\right)<0$ then the matrix $P\left(x_{*}\right)$ is required solution. This problem can be reduced to the minimization of a convex function under convex constraints.

Consider the following convex minimization problem

$$
\begin{aligned}
& \phi(x) \rightarrow \text { minimize. } \\
& \min _{\|v\|=1} v^{\mathrm{T}} P(x) v>0
\end{aligned}
$$

Let $X \subset \mathbb{R}^{n}$ be a convex set and $F: X \rightarrow \mathbb{R}$ be convex function. The vector $g \in \mathbb{R}^{n}$ is said to be a subgradient of $F(x)$ at $X_{*} \in X$ if for all $x \in X$

$$
F(x) \geq F\left(x_{*}\right)+g^{\mathrm{T}}\left(x-x_{*}\right) .
$$


The set of all subgradients of $F(x)$ at $x=x_{*}$ is denoted by $\partial F\left(x_{*}\right)$. If $x_{*}$ is an interior point of $X$ then the set $\partial F\left(x_{*}\right)$ is nonempty and convex. The following proposition follows from nondifferentiable optimization theory.

Proposition 1. Let $\phi(x)$ be defined as

$$
\phi(x)=\max _{y \in Y} f(x, y)
$$

where $Y$ is compact, $f(x, y)$ is continuous and differentiable in $x$. Then

$$
\partial \phi(x)=\operatorname{conv}\left\{\frac{\partial f(x, y)}{\partial x}: y \in Y(x)\right\}
$$

where $Y(x)$ is the set of all maximizing elements $y$ in (6), i.e.

$$
Y(x)=\{y \in Y: f(x, y)=\phi(x)\} .
$$

If for a given $x$ the maximizing element is unique, i.e. $Y(x)=\{y(x)\}$ then $\phi(x)$ is differentiable at $x$ and its gradient is

$$
\nabla \phi(x)=\frac{\partial f(x, y)}{\partial x}
$$

In the case of the Function (4)

$$
\begin{gathered}
\partial \phi(x)=\operatorname{conv}\left\{\frac{\partial}{\partial x}\left(u^{\mathrm{T}}\left(A_{i}^{\mathrm{T}} P+P A_{i}\right) u\right): i \text { maximizes } \lambda_{\max }\left(A_{i}^{\mathrm{T}} P+P A_{i}\right),\right. \\
u \text { is a corresponding unit eigenvector }\} .
\end{gathered}
$$

If for the given $x$ the maximizing $i$ is unique and $\lambda_{\max }\left(A_{i}^{\mathrm{T}} P+P A_{i}\right)$ is a simple eigenvalues, the differentiability of $\phi$ at the point $x$ is guaranteed [13].

We investigate problem (5) by Kelley's cutting-plane method.

This method converts the problem (5) to the problem

$$
\begin{aligned}
& c^{\mathrm{T}} z \rightarrow \min \\
& c_{1}(z) \geq 0, c_{2}(z) \geq 0,-1 \leq x_{i} \leq 1(i=1,2, \cdots, r)
\end{aligned}
$$

where $z=\left(x_{1}, x_{2}, \cdots, x_{r}, L\right)^{\mathrm{T}}, \quad c=(0,0, \cdots, 0,1)^{\mathrm{T}}, \quad c_{1}(z)=L-\phi(x), \quad c_{2}(z)=\min _{\|v\|=1} v^{\mathrm{T}} P v$.

Let $z^{0}$ be a starting point and $z^{0}, z^{1}, \cdots, z^{k}$ be $k+1$ distinct points.

At the $(k+1)$ th iteration, the cutting-plane algorithm solves the following LP problem

$$
\begin{array}{cc}
\text { minimize } & L \\
\text { subject to } & -h_{1}^{\mathrm{T}}\left(z^{0}\right) z \geq-h_{1}^{\mathrm{T}}\left(z^{0}\right) z^{0}-c_{1}\left(z^{0}\right) \\
-h_{2}^{\mathrm{T}}\left(z^{0}\right) z & \geq-h_{2}^{\mathrm{T}}\left(z^{0}\right) z^{0}-c_{2}\left(z^{0}\right) \\
\vdots \\
-h_{1}^{\mathrm{T}}\left(z^{k}\right) z & \geq-h_{1}^{\mathrm{T}}\left(z^{k}\right) z^{k}-c_{1}\left(z^{k}\right) \\
-h_{2}^{\mathrm{T}}\left(z^{k}\right) z & \geq-h_{2}^{\mathrm{T}}\left(z^{k}\right) z^{k}-c_{2}\left(z^{k}\right) \\
- & -1 \leq x_{i} \leq 1
\end{array}
$$

where $h_{j}\left(z^{i}\right)$ denotes a subgradient of $-c_{j}(z)$ at $z^{i}(i=1,2)$.

Let $z_{*}^{k}$ be the minimizer of the problem (8).

If $z_{*}^{k}$ satisfies the inequality $\min \left\{c_{1}\left(z_{*}^{k}\right), c_{2}\left(z_{*}^{k}\right)\right\} \geq-\varepsilon$, where $\varepsilon$ is a tolerance then $z_{*}^{k}$ is an approx- 
imate solution of the problem (7).

Otherwise define $j^{*}$ as the index for the most negative $c_{j}\left(z_{*}^{k}\right)$, update the constraints in (8) by including the linear constraint

$$
C_{j^{*}}\left(z^{k+1}\right)-h_{j^{*}}^{\mathrm{T}}\left(z^{k+1}\right)\left(z-z^{k+1}\right) \geq 0
$$

and repeat the procedure.

Recall that our aim is to find $x_{*}$ such that $P\left(x_{*}\right)>0$ and $\phi\left(x_{*}\right)<0$, but not the solution of the minimization problem (5), (7).

Theorem 2. If there exists $k$ such that

$$
c_{1}\left(z_{*}^{k}\right)>L^{k}, c_{2}\left(z_{*}^{k}\right)>0
$$

where $z_{*}^{k}=\left(x_{*}^{k}, L^{k}\right)$ is the minimizer of the problem (8), then the matrix $P=P\left(x_{*}^{k}\right)$ is a common solution to (3).

Proof:

$$
\begin{gathered}
\phi\left(x_{*}^{k}\right)=L^{k}-c_{1}\left(z_{*}^{k}\right)<0, \\
0<c_{2}\left(z_{*}^{k}\right)=\min _{\|v\|=1} v^{\mathrm{T}} P\left(x_{*}^{k}\right) v
\end{gathered}
$$

and by (5), $P\left(x_{*}^{k}\right)>0$ is a common solution to (3).

For the problem (5), (7) Kelley's method gives the following

\section{Algorithm 1.}

Step 1. Take an initial point $z^{0}=\left(x^{0}, L^{0}\right)^{\mathrm{T}}$. Compute $\phi\left(x^{0}\right)$ and $c_{2}\left(z^{0}\right)$. If $\phi\left(x^{0}\right)<0$ and $c_{2}\left(z^{0}\right)>0$ stop; otherwise continue.

Step 2. Determine $z_{*}^{k}$ by solving LP problem in (8). If $c_{1}\left(z_{*}^{k}\right)>L^{k}$ and $c_{2}\left(z_{*}^{k}\right)>0$ then stop; otherwise continue. Set $z^{k+1}=z_{*}^{k}$, update the constraints in (8) and repeat the procedure.

Example 1. Consider the switched system

$$
\dot{x} \in\left\{A_{1}, A_{2}, A_{3}\right\} x
$$

where

$$
A_{1}=\left(\begin{array}{ccc}
-2 & 5 & -6 \\
0 & -8 & 0 \\
-5 & -2 & -20
\end{array}\right), A_{2}=\left(\begin{array}{ccc}
-8 & 17 & -27 \\
9 & -44 & 27 \\
22 & -41 & -2
\end{array}\right) \text { and } A_{3}=\left(\begin{array}{ccc}
4 & 9 & -2 \\
-6 & -8 & 4 \\
1 & -10 & -6
\end{array}\right)
$$

are Hurwitz stable matrices.

Choose the initial point $z^{0}=\left(x_{1}^{0}, x_{2}^{0}, x_{3}^{0}, x_{4}^{0}, x_{5}^{0}, x_{6}^{0}, L^{0}\right)^{\mathrm{T}}=(1,0,0,1,0,1,1)^{\mathrm{T}}$, then

$$
P\left(x^{0}\right)=\left(\begin{array}{ccc}
1 & 0 & 0 \\
0 & 1 & 0 \\
0 & 0 & 1
\end{array}\right)
$$

$c_{1}\left(z^{0}\right)=-7.5247, \quad c_{2}\left(z^{0}\right)=1$ and $\phi\left(x^{0}\right)=\max _{i \in\{1,2,3\}} \lambda_{\max }\left(A_{i}^{\mathrm{T}} P\left(x^{0}\right)+P\left(x_{0}\right) A_{i}\right)=8.5247>0$.

We obtain $z^{1}=(-1,1,1,1,-1,1,-27.9933)^{\mathrm{T}}$ by solving LP problem in (8). Calculations give the following Table 1, and

$$
z^{15}=\left(x^{15}, L^{15}\right)^{\mathrm{T}}=(0.7811,0.6268,-0.1283,1,-0.1254,0.2383,-0.8206)^{\mathrm{T}} .
$$

Since $L^{15}-c_{1}\left(z^{15}\right)=-0.0287<0$ and $c_{2}\left(z^{15}\right)=0.2075>0$, 
Table 1. Kelley’s algorithm for Example 1.

\begin{tabular}{cccc}
\hline$k$ & $L^{k}$ & $c_{1}\left(z^{k}\right)$ & $c_{2}\left(z^{k}\right)$ \\
\hline 1 & -27.9933 & -209.7383 & -1.9999 \\
2 & -24.4038 & -127.1153 & -2.3326 \\
3 & -14.2596 & -106.2473 & -1.8092 \\
4 & -10.0497 & -63.4433 & -1.8878 \\
$\vdots$ & $\vdots$ & $\vdots$ & $\vdots$ \\
14 & -0.8465 & -1.1881 & 0.2694 \\
\hline
\end{tabular}

$$
P=P\left(x^{15}\right)=\left(\begin{array}{ccc}
0.7811 & 0.6268 & -0.1283 \\
0.6268 & 1 & -0.1254 \\
-0.1283 & -0.1254 & 0.2383
\end{array}\right)
$$

is a common positive definite solution for

$$
A_{i}^{\mathrm{T}} P+P A_{i}<0 \quad(i=1,2,3) .
$$

\section{Stable Member in a Polytope}

This part is devoted to the following question: Given a matrix family $\{A(q): q \in B\}$ where $B \subset \mathbb{R}^{l}$ is a compact, is there a stable matrix in this family?

In [12], a numerical algorithm has been proposed for a stable member in the affine matrix family $\left\{A(q): q \in \mathbb{R}^{l}\right\}$. In this algorithm the uncertainty vector $q$ varies in the whole space $\mathbb{R}^{l}$. On the other hand we consider the case where $q$ varies in a box $B \subset R^{l}$ and use the gradient algorithm for minimization of the nonconvex maximum eigenvalue function. By choosing appropriate step-size, we obtain the convergence.

Let $Z_{1}, Z_{2}, \cdots, Z_{r}\left(r=\frac{n(n+1)}{2}\right)$ be a basis for the subspace of $n \times n$ symmetric matrices and

$$
\begin{gathered}
Q_{i}(q)=\left(-Z_{i}\right) \oplus\left(A^{\mathrm{T}}(q) Z_{i}+Z_{i} A(q)\right), \\
\phi(x, q)=\lambda_{\max }\left(\sum_{i=1}^{r} x_{i} Q_{i}(q)\right)
\end{gathered}
$$

where $x=\left(x_{1}, x_{2}, \cdots, x_{r}\right)^{\mathrm{T}}, \quad q=\left(q_{1}, q_{2}, \cdots, q_{k}\right)^{\mathrm{T}}$.

Consider the problem

$$
\begin{aligned}
& \phi(x, q) \rightarrow \text { minimize. } \\
& \min _{\|x\|=1, q \in Q} v^{\mathrm{T}} P(x) v>0
\end{aligned}
$$

Theorem 3. There is a stable matrix in the family $A(q)$ if and only if $\phi^{*}=\min _{(x, q)} \phi(x, q)<0$.

Proof:

$$
\begin{gathered}
\phi^{*}<0 \Leftrightarrow \text { there exists }\left(x^{*}, q^{*}\right) \text { such that } \sum_{i=1}^{r} x_{i}^{*} Q_{i}\left(q^{*}\right)<0 \\
\Leftrightarrow\left(-\sum_{i=1}^{r} x_{i}^{*} Z_{i}\right) \oplus\left[A^{\mathrm{T}}\left(q^{*}\right)\left(-\sum_{i=1}^{r} x_{i}^{*} Z_{i}\right)+\left(-\sum_{i=1}^{r} x_{i}^{*} Z_{i}\right) A\left(q^{*}\right)\right]<0
\end{gathered}
$$




$$
\Leftrightarrow P\left(x^{*}\right)=\sum_{i=1}^{r} x_{i}^{*} Z_{i}>0 \text { and } A\left(q^{*}\right)^{\mathrm{T}} P\left(x^{*}\right)+P\left(x^{*}\right) A\left(q^{*}\right)<0
$$

By Lyapunov theorem, the matrix $A\left(q^{*}\right)$ is stable.

Example 2. Consider the family of matrices

$$
A(q)=A_{0}+q_{1} A_{1}+q_{2} A_{2}+q_{3} A_{3}, q_{1}, q_{2}, q_{3} \in[-1,1]
$$

where

$$
A_{0}=\left(\begin{array}{cccc}
1 & 0 & 2 & 0 \\
-2 & 0 & -3 & 0 \\
-5 & 1 & -1 & 0 \\
-3 & -1 & 0 & -2
\end{array}\right), A_{1}=\left(\begin{array}{cccc}
-2 & 0 & 3 & 0 \\
-1 & 0 & -3 & 2 \\
-3 & 3 & -1 & 0 \\
-4 & -1 & 0 & -2
\end{array}\right), A_{2}=\left(\begin{array}{cccc}
-1 & 0 & 2 & 0 \\
-3 & -1 & -3 & 0 \\
-3 & 2 & -1 & 2 \\
-2 & -1 & 0 & -2
\end{array}\right), A_{3}=\left(\begin{array}{cccc}
-1 & 0 & 0 & 1 \\
-1 & -2 & 3 & -2 \\
1 & -2 & 0 & -1 \\
0 & -2 & 1 & -5
\end{array}\right) .
$$

For $q=(0,0,0)^{\mathrm{T}}, A(q)=A_{0}$ is unstable. We apply the gradient algorithm to find a stable member in the family.

Let $x^{0}=\left(\frac{1}{2}, 0,0,0, \frac{1}{2}, 0,0, \frac{1}{2}, 0, \frac{1}{2}\right)^{\mathrm{T}}$ and $q^{0}=(1,0,0)^{\mathrm{T}}$. So

$$
a^{0}=\left(x^{0}, q^{0}\right)=\left(\frac{1}{2}, 0,0,0, \frac{1}{2}, 0,0, \frac{1}{2}, 0, \frac{1}{2}, 1,0,0\right)^{\mathrm{T}} .
$$

Then

$$
\begin{aligned}
\sum_{i=1}^{10} x_{i}^{0} Q_{i}\left(q^{0}\right) & =\left(\begin{array}{ccccccc}
-P\left(x^{0}\right) & \\
0 & A\left(q^{0}\right)^{\mathrm{T}} P\left(x^{0}\right)+P\left(x^{0}\right) A\left(q^{0}\right)
\end{array}\right) \\
& =\left(\begin{array}{ccccccccc}
-1 / 2 & 0 & 0 & 0 & 0 & 0 & 0 & 0 \\
0 & -1 / 2 & 0 & 0 & 0 & 0 & 0 & 0 \\
0 & 0 & -1 / 2 & 0 & 0 & 0 & 0 & 0 \\
0 & 0 & 0 & -1 / 2 & 0 & 0 & 0 & 0 \\
0 & 0 & 0 & 0 & -1 & 0 & 5 & 0 \\
0 & 0 & 0 & 0 & -3 & 0 & -6 & 2 \\
0 & 0 & 0 & 0 & -8 & 4 & -2 & 0 \\
0 & 0 & 0 & 0 & -7 & -2 & 0 & -4
\end{array}\right) .
\end{aligned}
$$

Maximum eigenvalue of this matrix and its corresponding unit eigenvector are

$$
\lambda_{\max }=2.1866, v=(0,0,0,0,0.7644,-0.4480,-0.1668,-0.4324)^{\mathrm{T}}
$$

respectively. Gradient of the function $\phi$ at $a^{0}$ is

$$
\left.\nabla \phi\right|_{a^{0}}=(-2.44,-1.86,-11.04,-2.78,1.93,7.50,4.30,2.52,7.46,2.35,0.28,0.50,-2.73)^{\mathrm{T}} .
$$

The first tencomponent of the vector $a^{1}=a^{0}-\left.t \cdot \nabla \phi\right|_{a^{0}}$ should be on the ten dimensional unit sphere. Therefore $t=0.01531$ and

$$
a^{1}=(0.53,0.02,0.16,0.04,0.47,-0.11,-0.06,0.46,-0.11,0.46,0.99,-0.007,0.04)^{\mathrm{T}} .
$$

After 4 steps, we get

$$
a^{4}=\left(x^{4}, q^{4}\right)=(0.59,0.03,0.04,0.009,0.41,-0.05,-0.04,0.49,-0.15,0.45,0.98,-0.03,0.08)^{\mathrm{T}}
$$


and $\phi\left(x^{4}, q^{4}\right)=-0.2585<0$. Therefore $A\left(q^{4}\right)$ is stable.

\section{Conclusion}

Two important problems from control theory are considered: the existence of common quadratic Lyapunov functions for switched linear systems and the existence of a stable member in a matrix polytope. We obtain new conditions which give new effective computational algorithms.

\section{References}

[1] Boyd, S. and Yang, Q. (1989) Structured and Simultaneous Lyapunov Functions for System Stability Problems. International Journal of Control, 49, 2215-2240. http://dx.doi.org/10.1080/00207178908559769

[2] Büyükköroğlu, T., Esen, Ö. and Dzhafarov, V. (2011) Common Lyapunov Functions for Some Special Classes of Stable Systems. IEEE Transactions on Automatic Control, 56, 1963-1967. http://dx.doi.org/10.1109/tac.2011.2137510

[3] Cheng, D., Guo, L. and Huang, J. (2003) On Quadratic Lyapunov Functions. IEEE Transactions on Automatic Control, 48, 885-890. http://dx.doi.org/10.1109/tac.2003.811274

[4] Dayawansa, W.P. and Martin, C.F. (1999) A Converse Lyapunov Theorem for a Class of Dynamical Systems Which Undergo Switching. IEEE Transactions on Automatic Control, 44, 751-760. http://dx.doi.org/10.1109/9.754812

[5] King, C. and Shorten, R. (2004) A Singularity Test for the Existence of Common Quadratic Lyapunov Functions for Pairs of Stable LTI Systems. Proceedings of the American Control Conference, Boston, 30 June-2 July 2004, 38813884.

[6] Mason, O. and Shorten, R. (2006) On the Simultaneous Diagonal Stability of a Pair of Positive Linear Systems. Linear Algebra and Its Applications, 413, 13-23. http://dx.doi.org/10.1016/j.laa.2005.07.019

[7] Narendra, K.S. and Balakrishnan, J. (1994) A Common Lyapunov Function for Stable LTI Systems with Commuting A-Matrices. IEEE Transactions on Automatic Control, 39, 2469-2471. http://dx.doi.org/10.1109/9.362846

[8] Shorten, R.N. and Narendra, K.S. (2002) Necessary and Sufficient Conditions for the Existence of a Common Quadratic Lyapunov Function for a Finite Number of Stable Second Order Linear Time-Invariant Systems. International Journal of Adaptive Control and Signal Processing, 16, 709-728. http://dx.doi.org/10.1002/acs.719

[9] Shorten, R.N., Mason, O., Cairbre, F.O. and Curran, P. (2004) A Unifying Framework for the SISO Circle Criterion and Other Quadratic Stability Criteria. International Journal of Control, 77, 1-8. http://dx.doi.org/10.1080/00207170310001633321

[10] Liberzon, D. and Tempo, R. (2004) Common Lyapunov Functions and Gradient Algorithms. IEEE Transactions on Automatic Control, 49, 990-994. http://dx.doi.org/10.1109/tac.2004.829632

[11] Polyak, B.T. and Shcherbakov, P.S. (2005) Hard Problems in Linear Control Theory: Possible Approaches to Solution. Automation and Remote Control, 66, 681-718. http://dx.doi.org/10.1007/s10513-005-0115-0

[12] Polyak, B.T. and Shcherbakov, P.S. (1999) Numerical Search of Stable or Unstable Element in Matrix or Polynomial Families: A Unified Approach to Robustness Analysis and Stabilization. Robustness in Identification and Control Lecture Notes in Control and Information Sciences, 245, 344-358. http://dx.doi.org/10.1007/bfb0109879

[13] Horn, R.A. and Johnson, C.R. (1985) Matrix Analysis. Cambridge University Press, Cambridge. 
Scientific Research Publishing (SCIRP) is one of the largest Open Access journal publishers. It is currently publishing more than 200 open access, online, peer-reviewed journals covering a wide range of academic disciplines. SCIRP serves the worldwide academic communities and contributes to the progress and application of science with its publication.

Other selected journals from SCIRP are listed as below. Submit your manuscript to us via either submit@scirp.org or Online Submission Portal.
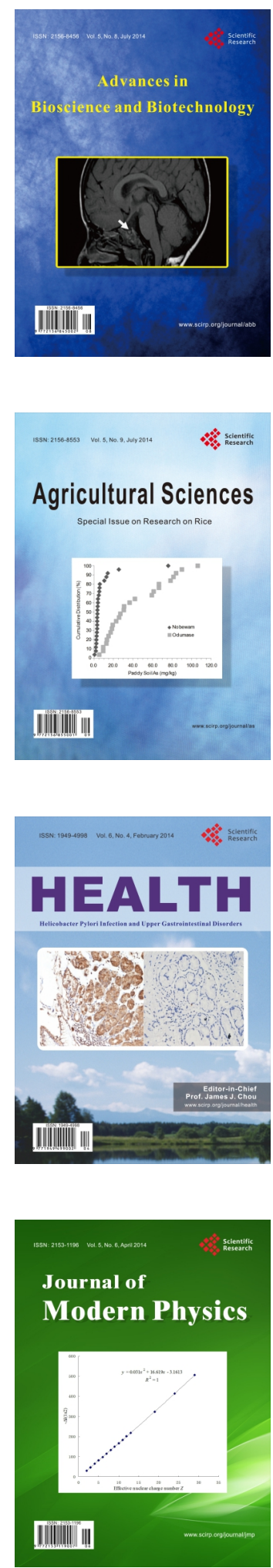
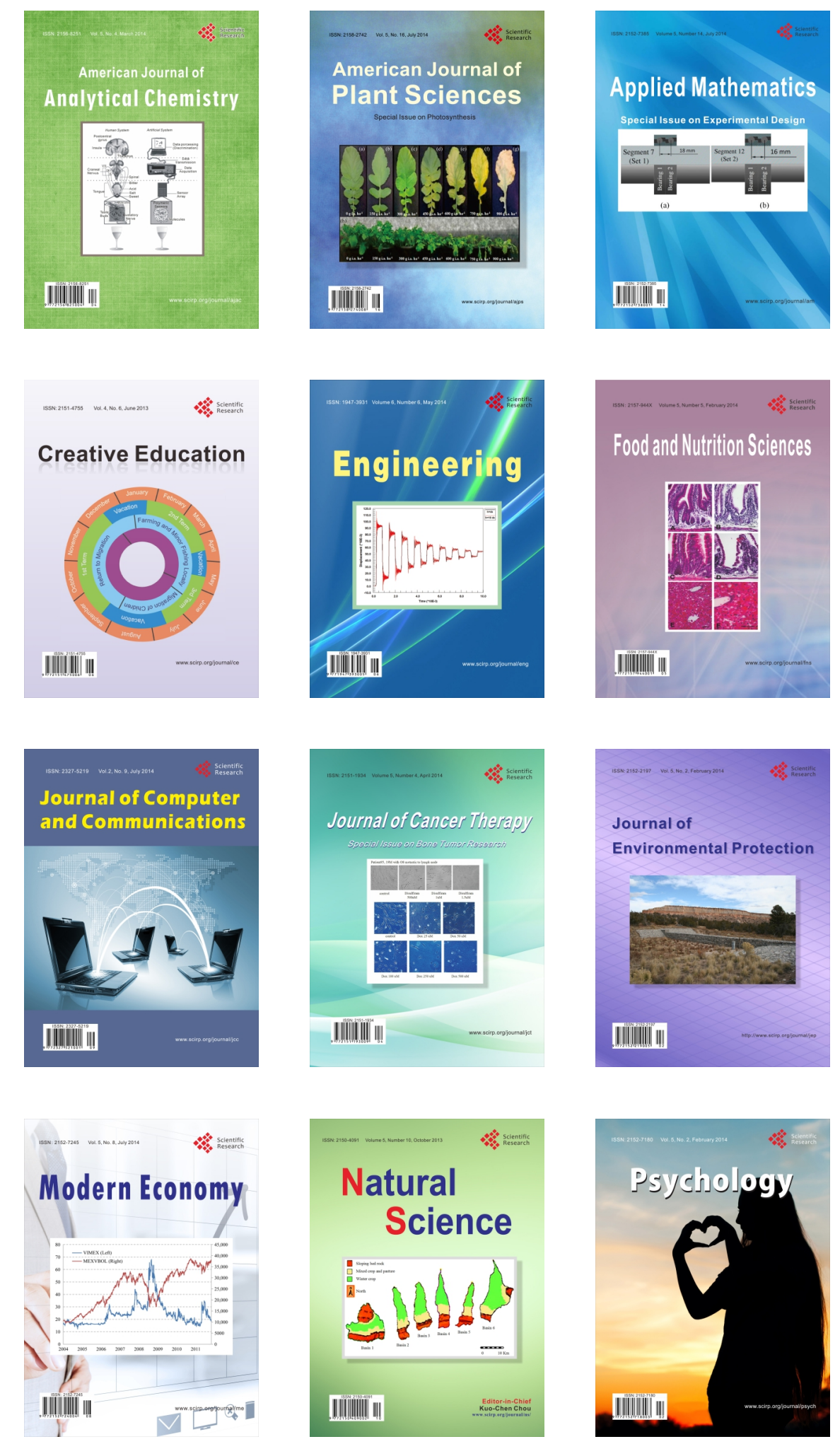